\title{
Turismo e Voluntariado: um estudo sobre as publicações em revistas científicas nacionais e internacionais
}

\section{Tourism and Volunteering: a study about the publications in national and international scientific journals}

\section{Turismo y Voluntariado: un estudio de las publicaciones en revistas científicas nacionales y internacionales}

Renê Corrêa do Nascimento ${ }^{1}$

\begin{abstract}
Resumo
Este estudo apresenta análise exploratória e crítica dos entendimentos sobre a temática do Turismo e Voluntariado, possibilitando maiores esclarecimentos a respeito da prática do turismo associado ao trabalho voluntário. O corpus da pesquisa resultou da busca, captação e análise de artigos de periódicos publicados em revistas científicas internacionais e nacionais, constatando-se, a respeito das últimas, que não houve produção nas principais revistas científicas brasileiras que abordam as temáticas relacionadas ao Turismo e Voluntariado. Por seu caráter exploratório e qualitativo, a presente pesquisa não é conclusiva, porém, fundamental para melhor posicionamento sobre a questão do turismo associado ao trabalho voluntário. Ressalta-se, ainda, que a temática deste trabalho é ampla e abrange áreas diversas, proporcionando, assim, substrato para entendimento de outros horizontes ligados tanto à temática do trabalho voluntário quanto ao turismo e suas diversificações em termos de novas modalidades de viagens e experiências proporcionadas.
\end{abstract}

Palavras-Chave:turismo e hospitalidade; voluntariado;turismo voluntário;produção científica internacional;artigosde periódicos.

\section{Abstract}

This study presents a critique exploratory analysis of understandings on the subject of Tourism and Volunteering, providing further information about the practice of tourism associated to the volunteer work. The corpus of the research is a result of the search, capture and analysis of journal articles published in international and national journals. The research found that there was no production on the theme in the main Brazilian scientific journals that address the issues related to tourism. Due to the exploratory and qualitative character of the present study, it is not conclusive, however, fundamental to a better position on the issue of tourism associated with volunteer work. It should be noted also that the theme of this work is broad and covers several areas, thus providing substrate for understanding

\footnotetext{
${ }^{1}$ Professor Doutor do Curso de Turismo da Escola de Comunicações e Artes da Universidade de São Paulo e Professor Titular do Programa de Mestrado em Hospitalidade da Universidade Anhembi Morumbi.
} 
other horizons linked to both the theme of volunteering and tourism and its diversification in terms of new modes of travel and experiences proportionated.

Keywords:tourism and hospitality;volunteering;volunteer tourism;international scientific production;journal articles.

\section{Resumen}

Este estudio presenta un análisis exploratorio y crítico de los entendimientos sobre la temática del Turismo y Voluntariado, que permite aclarar aspectos sobre la práctica del turismo asociado al trabajo voluntario. El corpus de la investigación resulta de la búsqueda, captación y análisis de artículos publicados en revistas científicas internacionales y brasileñas, constatándose respecto a las últimas, que no hubo producción en las principales revistas cientificas brasileñas que abarcaran las temáticas relativas al Turismo y Voluntariado. Por su carácter exploratorio y cualitativo, la presente investigación no es conclusiva, sin embargo es fundamental para un mejor posicionamiento sobre la cuestión del turismo asociado al trabajo voluntario. Se resalta aun que la temática de este trabajo es amplia y abarca áreas diversas, proporcionando así un sustracto para la comprehensión de otros horizontes conectados tanto a la temática del trabajo voluntario como al turismo y sus diversificaciones en términos de nuevas modalidades de viajes y experiencias proporcionadas.

Palabras Clave:turismo y hospitalidade;voluntariado;turismo voluntario;producción científica internacional; artículos de periódicos.

\section{Introdução}

As pesquisas em Turismo, hoje, buscam investigar as múltiplas realidades sociocultural, ambiental e econômica e apresentar as possibilidades de complexas e amplas variáveis, que podem proporcionar uma consolidação da atividade nos mais diferentes cenários. Sabe-se que no paradigma tradicional da oferta turística, prevalecem sobremaneira os estudos que investigam o produto material, amplamente utilizado na atividade. Entretanto, questões referentes à cultura imaterial tornam-se cada vez mais significativas e urgentes no corolário de opções possíveis, considerando a velocidade das transformações advindas do processo de globalização que, se por um lado busca a homogeneização e padronização dos usos e costumes, por outro carrega em si a própria contraditoriedade, quando se vislumbra a emergência das identidades locais e particulares que se materializam, em especial, nas culturas imateriais. 
Nesse sentido, permite-se uma observação que se baseia na ideia, na emoção e na constatação a partir da experiência do autor como docente, pesquisador e observador do ser humano e, mormente, do turismo: o turismo pode ser o qualificativo de uma "filosofia do ser", posto que também alberga em seu bojo as atitudes de vida, as condutas eleitas, a ética global, a defesa da vida e do meio ambiente, e a defesa e exercício de liberdades; conduz a uma busca permanente de agregação mundial e livre trânsito de todos os habitantes do planeta, atitudes fraternas e solidárias, para dizer o mínimo de sua expressão manifesta; mas nele ainda estão presentes os sentimentos, as motivações individuais, a relação fraterna e hospitaleira, os sonhos, as ilusões, os imaginários pessoais e coletivos, o gosto do inusitado apreendido, a procura da felicidade.

Nesse contexto situa-se o turismo voluntário entendido como as viagens turísticas que aliam a prestação de serviços voluntários dirigidos às causas sociais com outras motivações turísticas, interagindo de forma ativa em uma "experiência de vida".

Tradicionalmente, o trabalho voluntário se desenvolveu mais por razões religiosas voltadas, principalmente, à caridade. Numa visão tradicional e limitativa, o voluntariado caracterizavase apenas pelo ato de "dar apenas por dar", reforçando-se assim, num processo histórico, a construção maior dos vínculos de dependência entre ricos e pobres, patrões e empregados, assistidos e voluntários.

Essas antigas e tendenciosas concepções de voluntariado, como ações de caridade, como assistencialismo, em vista de muitos paradigmas da globalização, estão superadas.Na atualidade, o voluntariado vai além de uma simples ação de caridade, busca a qualificação da sua atuação e a otimização dos possíveis recursos disponíveis, com o intuito de ajudar efetivamente as mudanças de valores e práticas, ou divulgar informações que construam uma base sustentável para as comunidades e os atendidos na ação voluntária.

Em termos conceituais, a Associação Internacional de Esforço Voluntário (IAVE), em setembro de 1990, em Paris, redigiu uma Declaração Mundial do Voluntariado, definindo-o como sendo "um serviço comprometido com a sociedade e alicerçado na liberdade de escolha. O voluntariado promove um mundo melhor e torna-se um valor para todas as sociedades"(Portal do voluntário, 2008).

Acredita-se que o princípio no qual se rege o voluntariado é o cuidado, no qual se moldam as 
necessidades humanas não superficiais, contradizendo aos valores vividos na atualidade. Quanto a isto, "do ponto de vista existencial, o cuidado se acha, a priori, antes de toda atitude e situação do ser humano, o que sempre significa dizer que ele se acha em toda atitude e situação de fato" (Heidegger, apud Boff, 2003, p.35). O cuidado é entendido aqui como uma maneira de se conhecer, e de reconhecer as necessidades alheias: é uma ação com a realidade, é permanentemente atrelado ao ser humano.

O trabalho voluntário procura entrar em sintonia com a comunidade e o meio ambiente e, assim, agir integrado junto a estes. Numa perspectiva de mudanças de valores, permite ao ser humano viver a experiência fundamental daquilo que tem importância e realmente faz diferença, proporcionando o desenvolvimento de atitudes como o respeito, a reciprocidade, a igualdade, entre outras.

Tendo como preocupação central estudar o turismo voluntário, buscou-se conhecer a literatura cientifica publicada no Brasil, consultando-se os principais periódicos científicos da área de Turismo e Hospitalidade classificados na lista Qualis em 2011 pela Capes - Coordenação de Aperfeiçoamento de Pessoal de Nível Superior: Turismo em Análise; Turismo - Visão \& Ação; Caderno Virtual de Turismo; Revista Acadêmica Observatório de Inovação do Turismo; Patrimônio: Lazer \& Turismo; Revista de Estudos Turísticos, Revista Global Tourism e Revista Turismo \& Desenvolvimento. Surpreendentemente não foram identificados artigos sobre "turismo e voluntariado", "turismo voluntário" ou "voluntarismo", ao contrário de periódicos internacionais que têm publicado frequentemente artigos sobre o tema.

Por esta razão, este artigo apresenta os resultados de uma pesquisa sobre a produção científica referente ao tema "Turismo e Voluntariado", a fim de caracterizar e analisar os artigos publicados em periódicos científicos internacionais. Trata-se de uma pesquisa exploratória, de caráter documental e desenvolvida com base na análise de conteúdo. Os dados foram coletados em duas bases de dados multidisciplinares com ampla cobertura na área das Ciências Sociais Aplicadas, na qual se enquadram os estudos de Turismo: a Web of Science $(\mathrm{WOS})^{2}$ e a SciVerse Scopus ${ }^{3}$.

\footnotetext{
${ }^{2}$ A Web of Science (WOS), disponibilizada pelo grupo Thomson Reuters, é um indexador de citações que abrange 12 mil dos periódicos científicos de grande impacto do mundo, com ampla cobertura cronológica, possuindo registros de artigos, reviews e proceedings papers desde os anos de 1900.
} 
Ambas as bases são relevantes para estudos da produção científica internacional sobre o Turismo, ainda que existam pesquisas (FALAGAS et al, 2007; NORUZI, 2005) que apontem uma defasagem destes indexadores em relação ao Google Scholar, base de dados gratuita fornecida pelo Google.Apesar da discussão em relação à adequação das bases de dados em relação à cobertura de dados e repercussões principalmente no campo da bibliometria, destaca-se a importância da produção científica publicada em periódicos científicos virtude do seu caráter atualizado, além do acesso facilitado pela internet.

Destaca-se ainda que o tema central deste trabalho não possui nomenclatura consensual no meio acadêmico brasileiro por se tratar de um nicho recentemente abordado nos estudos sobre o turismo (inclusive em nível internacional). Dessa forma, também se justifica a intenção de se explorar os entendimentos sobre a temática, possibilitando maiores esclarecimentos a respeito da prática do turismo (principalmente em termos de viagens internacionais) associado ao trabalho voluntário, modalidadeem crescente popularidade.

\section{Metodologia}

Os artigos foram levantados nas bases de dados acima citadas, a partir de buscas nos campos "título", "abstract" e "palavra-chave",intuindo-se captar artigos que abordassem o tema "Turismo e Voluntariado", realizadas durante o mês de outubro de 2010 (1 ${ }^{\mathrm{a}}$ quinzena) e durante o mês de fevereiro de 2012.

Inicialmente acessaram-se as páginas de pesquisa de Web of Science $^{4}$ e do Scopus $^{5}$ para prospecção de resultados relacionados à temática "Turismo e Voluntariado". Em seguida procedeu-se o armazenamento (download) dos abstractsde artigos selecionados, e por fim o armazenamento dos artigos completos.

A seleção das palavras-chave para pesquisa no WOS e Scopus foi pautada pela relevância de termos dentro da área de pesquisa do "Turismo e Voluntariado", ou seja: volunteer tourism,

\footnotetext{
${ }^{3}$ A SciVerse Scopus, fornecido pela Elsevier, é a maior base de dados bibliográficos, com mais de 19,5 mil títulos de periódicos, tais como "Tourism Management", "Annals of Tourism Research", além de mais de 5 mil editores internacionais, como Elsevier, Butterworths e Channel View Publications.

${ }^{4}$ Disponível em: $<$ http://thomsonreuters.com/products_services/science/science_products/az/web of science/\#tab1 $>$.

${ }^{5}$ Disponível em:<http://www.info.sciverse.com/scopus/about $>$.
} 
voluntary e voluntourism. Além dessas palavras-chave, foram incluídas pesquisas combinando dois e três termos: voluntary AND tourism, volunteer tourism AND sustainability, voluntary AND tourism AND sustainability. As pesquisas com mais de um termo-chave foram relacionadas pelo conectivo $A N D$ para que houvesse resultados que possivelmente abordariam a temática como tema central. A opção por incluir palavras de significado e uso amplo comovoluntary, tourism e sustainabilityintuiu possibilitar a abrangência dos estudos que abordam o Turismo Voluntariado para além das publicações especializadas em Turismo/ Lazer/ Hospitalidade.

Passou-se então a se verificar a temática principal do artigo, identificando-a por meio da análise do título do artigo e o do título do periódico no qual o artigo fora publicado. No caso do título ser amplo, assim não possibilitando a identificação de uma temática central, passouse a analisar o abstract e as palavras-chave para identificar sua relevância no tema. Por fim os artigos selecionados foram separados em "pertinentes" e "não pertinentes" à temática “Turismo e Voluntariado", ou seja, artigos constituindo a amostra final da pesquisa.

Os resultados obtidos são apresentados a seguir nos tópicos de caracterização geral dos artigos, apresentando aspectos da amostra inicial à final, e nas concepções sobre o tema, explorando o conteúdo dos documentos.

\section{Produção Científica Internacional sobre Turismo e Voluntariado}

Caracterização geral dos artigos

De um total de 277 artigos levantados inicialmente, 122 apresentaram resultados duplicados, o que levou a 155 artigos publicados no período de 1979 a 2012 como amostra inicial desta pesquisa, conforme apresentado na tabela 1.Considerando as duas bases de dados, tem-se que a maioria (64\%) encontra-se na Scopus e a minoria (36\%) na WOS (figura 1). Esse resultado indica maior cobertura de artigos sobre turismo voluntário na base Scopus, o que já era esperado tendo em vista a abrangência da mesma e em razão do tema ser recente na literatura especializada em Turismo. 
Tabela 1 - Amostra inicial de artigos sobre Turismo e Voluntariado publicados em periódicos da Web of Science e Scopus

\begin{tabular}{|c|c|c|c|c|c|}
\hline \multirow{2}{*}{ Termo isolado } & \multirow{2}{*}{$\begin{array}{c}\text { Termo } \\
\text { Associado } 1\end{array}$} & \multirow{2}{*}{$\begin{array}{c}\text { Termo } \\
\text { Associado } 2\end{array}$} & \multirow{2}{*}{ Fonte } & \multicolumn{2}{|c|}{ Frequência } \\
\hline & & & & $\left(\mathbf{n}^{0}\right)$ & $(\%)$ \\
\hline $\begin{array}{l}\text { Volunteer } \\
\text { Tourism }\end{array}$ & - & - & WOS & 28 & 10,10 \\
\hline $\begin{array}{l}\text { Volunteer } \\
\text { Tourism }\end{array}$ & - & - & SCOPUS & 42 & 15,17 \\
\hline Voluntourism & - & - & WOS & 3 & 1,08 \\
\hline Voluntourism & - & - & SCOPUS & 6 & 2,16 \\
\hline Voluntary & (AND) Tourism & - & WOS & 61 & 22,03 \\
\hline Voluntary & (AND) Tourism & - & SCOPUS & 112 & 40,43 \\
\hline $\begin{array}{l}\text { Volunteer } \\
\text { Tourism }\end{array}$ & $\begin{array}{c}(A N D) \\
\text { Sustainability }\end{array}$ & - & WOS & 1 & 0,37 \\
\hline $\begin{array}{l}\text { Volunteer } \\
\text { Tourism }\end{array}$ & $\begin{array}{c}(A N D) \\
\text { Sustainability }\end{array}$ & - & SCOPUS & 4 & 1,44 \\
\hline Voluntary & (AND) Tourism & $\begin{array}{c}(A N D) \\
\text { Sustainability }\end{array}$ & WOS & 7 & 2,53 \\
\hline Voluntary & (AND) Tourism & $\begin{array}{c}(A N D) \\
\text { Sustainability }\end{array}$ & SCOPUS & 13 & 4,69 \\
\hline SUBTOTAL (sen & emover resultados & uplicados) & & 277 & $100 \%$ \\
\hline TOTAL (sem res) & ados duplicados) & & & 155 & $72 \%$ \\
\hline
\end{tabular}

Fonte: Elaboração própria, 2012. 


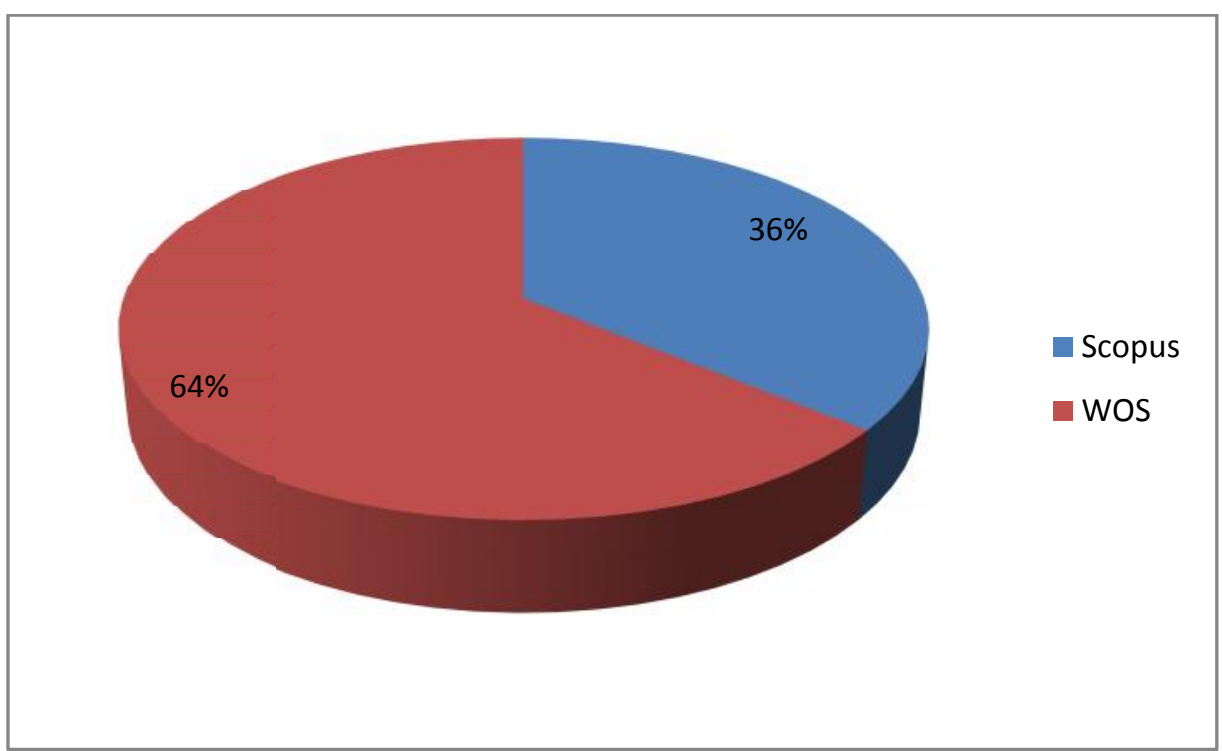

Figura 1 - Distribuição dos artigos sobre Turismo e Voluntariado nas bases de dados internacionais - 1979 a 2012

Fonte: Elaboração própria, 2012.

Ressalta-se o baixo aproveitamento dos resultados obtidos pelas buscas que utilizaram as palavrasVoluntary AND Tourismem virtude de:

a) A busca captou o uso da palavravoluntary de maneira independente de turismo, apesar de ambas constarem no abstract dos resultados obtidos. Assim, o significado devoluntaryfoi utilizado como "contribuição [monetária] voluntária", "diminuição [de emissões de gases de efeito estufa, de impacto ambiental por parte dos habitantes locais] voluntária", “colaboração voluntária da comunidade local” etc., Em relação ao termotourism, os resultados apresentaram apenas uma menção ao fenômeno turístico, não o abordando como temática central, como por exemplo, ao apenas citar o turismo como um reflexo econômico em virtude de um meio ambiente de rico em biodiversidade (Aulong, Figuieres e Thoyer, 2011).

b) A contagem dos artigos aproveitados que constaram em mais de uma busca não foi registrada de maneira duplicada, o que diminuiu o aproveitamento paraVoluntary ANDTourism, por serem termos menos específicos que os demais, os quais foram priorizados para contagem. 
Nota-se que o tema ainda não possui uma nomenclatura consensual na literatura internacional, sendo comumente relacionado como "subtipo" de outras modalidades mais trabalhadas no meio acadêmico, tais como: Ecoturismo, Turismo Social e Turismo Alternativo.A maioria dos autores emprega o termovoluntary associando-o atourism em seus artigos (173, 62,45\%), seguido porvolunteer tourism(75, 27,08\%) evoluntary and tourism and sustainability (20, $7,23 \%)$; poucos empregam o termovoluntourism $(9,3,24 \%)$, cujo uso e recente, apresentandose somente a partir de 2009.

Dentro da amostra de 155 artigos internacionais, 67 (43,23\%) foram publicados em periódicos cujo tema principal é o Turismo/Lazer/Hospitalidade e 88 (56,77\%) eram provenientes de periódicos sobre temas diversos, como por exemplo, Ecologia e Políticas Públicas entre outras.

Ainda como forma de delimitar o escopo deste trabalho, os 155 artigos foram filtrados para selecionar os que tratavam do "Turismo e Voluntariado" como tema central (tabela 2). Foram considerados pertinentes ao estudo 45 artigos (29\%), sendo 10 publicados em periódicos de temáticas diversas, entre elasEnvironmental Management,Biological Conservation eService Industries Journal; e 35 provenientes de periódicos específicos de Turismo/Lazer/Esporte. Nestas ultimas sobressaem os artigos publicados no "Journal of Sustainable Tourism" (9), seguidos pelos do "Annals of Tourism Research" (5) e "Tourism Management" (4); os demais apresentam 1 ou 2 artigos sobre o tema.Esse resultado mostra que o turismo voluntariado na literatura internacional se aproxima mais, como já citado, as questões de sustentabilidade do turismo.

Tabela 2 - Amostra final de artigos sobre Turismo e Voluntariado por titulo de periódico

\begin{tabular}{l|c}
\hline \multicolumn{1}{c|}{ Título do Periódico } & Frequência de Artigos $\left(\mathbf{n}^{\mathbf{0}}\right)$ \\
\hline Anatolia & 1 \\
\hline Annals of Tourism Research & 5 \\
\hline Asia Pacific Journal of Tourism Research & 2 \\
\hline Biological Conservation & 1 \\
\hline Current Issues in Tourism & 2 \\
\hline Environmental Management & 1 \\
\hline
\end{tabular}




\begin{tabular}{l|l}
\hline European Journal of Development Research & 1 \\
\hline Geoforum & 1 \\
\hline Globalization and Health & 1 \\
\hline International Journal of Tourism Research & 2 \\
\hline Journal of Ecotourism & 1 \\
\hline Journal of International Development & 1 \\
\hline Journal of Leisure Research & 1 \\
\hline Journal of Sport and Tourism & 9 \\
\hline Journal of Sustainable Tourism & 2 \\
\hline Journal of Travel \& Tourism Marketing & 2 \\
\hline Leiure Sciences & 1 \\
\hline Loisir et Société & 1 \\
\hline Prace i Studia Geograficzne & 1 \\
\hline Servie Industries Journal & 1 \\
\hline Services Marketing Quarterly & 1 \\
\hline Tourism & 1 \\
\hline Tourism Geographies & 3 \\
\hline Tourism Management & 1 \\
\hline Tourismos & $\mathbf{4 5}$ \\
\hline Total & \\
\hline
\end{tabular}

Fonte: Elaboração própria, 2012.

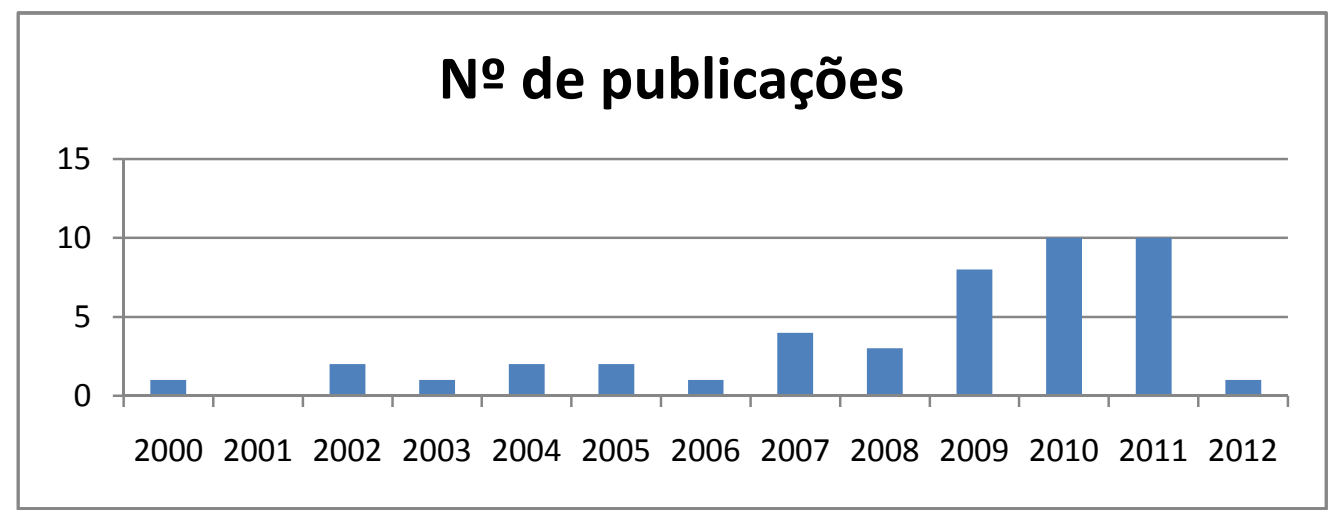

Figura 2- Artigos sobre Turismo e Voluntariado por ano de publicação - 2000 a 2012

Fonte: Elaboração própria, 2012. 
Distribuindo esses artigos por ano de publicação (figura 2), verifica-se que o tema aparece nos periódicos somente a partir de 2000, com maior incidência a partir do final dessa década. Obviamente, a produção em 2012 é parcial, uma vez que foi levantada em fevereiro desse ano, e poderá apresentar uma ascendência significativa até o final da década.

\section{Concepções Temáticas}

A temática "Turismo e Voluntariado", comumente denominadavolunteer tourismna literatura internacional, em linhas gerais é o termo aplicado "àqueles turistas que, por vários motivos, voluntariam de maneira organizada para empreender férias que envolvam auxiliar ou aliviar a pobreza material de alguns grupos da sociedade, restabelecer certos ecossistemas ou pesquisar aspectos da sociedade ou do meio ambiente natural" (WEARING,2001).

Tal descrição vem ao encontro do que foi observado como concepção de "Turismo e Voluntariado" pelos 45 artigos selecionados para este estudo, os quais ressaltam, sobretudo, essa forma de turismo como alternativo,entendido, em geral (uma vez que, também, não há uma definição consensual sobre esse tipo de turismo), como diferente do turismo de massa/convencional, ressaltando-se a maior possibilidade de envolvimento entre a comunidade receptora e o turista (PEARCE, 1992).

Em relação à discussão teórica sobre o "Turismo e Voluntariado", nota-se que a maioria dos posicionamentos ressalta-o como benéfico tanto para as comunidades que recebem os turistas/voluntários como para os próprios turistas, com poucos questionamentos em relação aos aspectos negativos que poderiam ser relacionados à prática do turismo associado ao trabalho voluntário(Wearing e Neil,2000; McGehee,2002; Piotrowska, 2003; Galley e Clifton,2004; McGehee e Santos, 2005; Campbell e Smith, 2006; McIntosh e Zahra, 2007; Harlow e Pomfret, 2007; Theerapappisit, 2009; Bailey e Russell, 2010).

O trabalho de McGehee e Santos (2005), por exemplo, ao estudar três organizações americanas de trabalho voluntário (Mobility International, WorldPULSE e The AmericanJewish World Service), após coleta de dados através da metodologia de focus groups, destaca que a participação de voluntários resulta num efeito positivo de suporte às causas de um projeto. Os autores destacam a associação do turismo ao trabalho voluntário 
como benéfico, no sentido em que produz um "fértil substrato para o desenvolvimento de redes de contato e aumento da consciência" em relação às temáticas de um determinado projeto.

Entre aqueles que não se aprofundam em discutir os pontos negativos da prática, há autores que se detiveram a analisar os aspectos mercadológicos, tais como a rentabilidade (Brightsmith, 2010;Brightsmith, Stronza e Holle, 2008)e a possibilidade de ampliação do mercado de oferecimento de programas de Turismo associado ao trabalho voluntário (Benson e Henderson, 2011; Wymer e Findley, 2010; Brumbaugh, 2010; Blackman e Benson,2010).

No trabalho de Bringhtsmigh,Stronza e Holle (2008) foram estudados meios de financiamento de projetos de pesquisa em conservação ambiental envolvendo a parceriaentre a The Tambopata Macaw Project, um projeto de pesquisa,a Rainforest Expeditions, uma operadora de Ecoturismo, e aThe Earthwatch Institute, uma ONG de recrutamento de voluntários. Nesse trabalho, destacou-se o turismo associado ao trabalho voluntário como uma fonte potencial e relevante de financiamento, pois, enquanto os cientistas da área de conservação e agências de recrutamento (tal qual a The Earthwatch) desenvolvem os projetos, os voluntários proporcionam os recursos financeiros e a própria força de trabalho em si. Os resultados em relação a essa tripla parceria mostraram-se vantajosos: com a interação com os voluntários, proporcionado pela The Earthwatch, os fundos totalizaram mais de US\$ 400.000,00 em dinheiro, benefícios e serviços, o que proporcionou a continuidade do projeto durante sete anos sem interrupções. Além dos benefícios para o projeto,a operadora e a própria recrutadora de voluntários puderam ser beneficiadas financeiramente.

Ainda sem levantar questionamentos quanto ao fenômeno do "Turismo e Voluntariado", o trabalho de Benson e Henderson (2011) faz uma análise do mercado turístico associado ao trabalho voluntário, cuja coleta de informações foi feita por meio de questionários, entrevistas e observações. O fio condutor da análise foi baseado no modelo das cinco forças de Porter (1980) e na perspectiva do SPACE (Strategic Position and Action Evaluation). Como resultado da análise, os autores concluíram que, apesar das organizações desse setor apresentarem a habilidade para contribuir com a sustentabilidade (ambiental), os riscos em operar no setor são relativamente altos, ainda que nenhuma das cinco forças de Porter tenham 
apontado potencial suficiente para afetar os lucros e, assim, o balanço da empresa seria satisfatório.

Como nota Guttentag (2009), o grande otimismo inspirado pelo crescente interesse dos turistas em dedicar as férias para realizar trabalhos voluntários associando-os a uma viagem tem sido acompanhado por uma literatura acadêmica pouco crítica a respeito dessa modalidade de turismo. Entre os pontos positivos mais elucidados na literatura sobre o assunto, Guttentag (idem) destaca: os próprios resultados alcançados pelo trabalho, a receita gerada para a comunidade local (ou organização que organiza o trabalho voluntariado), a conservação ambiental, o crescimento pessoal do participante voluntário e a experiência intercultural.

Segundo o mesmo autor, os aspectos positivos são acompanhados por uma literatura que se reduz a identificar o perfil dos viajantes e investigar suas motivações para participar de uma viagem de turismo voluntariado. Nesse sentido, destacam-se na amostra 12 artigos direcionados à observação do perfil e das motivações dos turistas (Galley e Clifton, 2004; Campbell e Smith, 2006; McIntosh e Zahra, 2007; Graye Campbell, 2007; Harlowe Pomfret, 2007; Coghlan, 2007; Raymond e Hall, 2008; Ooi e Laing, 2010; Brumbaugh, 2010; Lo e Lee,2011; Jarvis e Blank, 2011; Chen e Chen, 2011).

Apesar dos diversos trabalhos em que predominam a dissertação sobre os aspectos positivos na relação turismo e voluntariado, há artigos que se destacaram em virtude de um posicionamento crítico a respeito do fenômeno. O trabalho de Simpson (2004) estuda a experiência oferecida pelogap year ${ }^{6}$,especificamente aquelas relacionadas ao trabalho voluntário em países do "terceiro mundo", com foco nos programas de curta duração (de menos de 6 meses), nos quais participam jovens entre 18 e 20 anos. A autora estuda o reflexo desses tipos de projetos (experiências) na construção de um entendimento sobre o desenvolvimento, combinando uma análise etnográfica com a análise do material promocional dogap year, sendo a amostra para a análise etnográfica composta por estudantes que estavam

\footnotetext{
${ }^{6}$ Segundo definição do site $<$ http://www.gapyear.com/>., gap year é uma experiência, geralmente realizada após o término do ensino secundário, com duração variável, na qual se propõe viagens internacionais para a complementação do currículo do viajante com experiências não convencionais, além da interação como outras culturas. As viagens do Gap Year podem ser, ou não, para realização de trabalhos voluntários em comunidades pobres.
} 
desenvolvendo trabalhos voluntários com crianças e que passariam de 3 a 6 meses na América do Sul.

Nesse estudo Simpson (2004) realiza entrevistas com perguntas abertas além da coleta de informações em sites e materiais promocionais como método para adquirir substrato para a análise qualitativa. Observa a falta nos programas degap year de uma "pedagogia sobre justiça social", a qual evitaria a construção de estereótipos e simplificação das diferenças sociais, econômicas e sobre o entendimento sobre o desenvolvimento. Destacou o fato da "indústria degap year" promover uma imagem "do outro terceiro mundo", ou seja, uma separação entre "nós [ricos] e eles [pobres]", reforçada por "clichês" promovidos nos sites e outras formas de comunicação, mensagens estas amparadas sobre o valor das "boas intenções do ocidente". Assim, a autora disserta que a "face pública" do desenvolvimento construída no gap year acaba se tornando o "quadro" que delimita a experiência do voluntário viajante, proporcionando a este uma visita ao "terceiro mundo" onde as desigualdades sociais são frutos da sorte, ou onde as mudanças ocorrerão por intervenção de estrangeiros com pouca qualificação, além da ausência da experiência compartilhada entre as pessoas.

Simpsom (2004) conclui que a "indústria de gap year" opera num sistema de desigualdade, no qual fica bem estabelecido quem "é" o desenvolvimento, simplificado como algo "factível". Nesse sentido, recomenda o emprego de uma orientação voltada à justiça social (entendida simplificadamente como o reconhecimento da desigualdade social) para que não haja uma dissimulação da desigualdade, mas sim que as viagens para trabalho voluntário se enquadrem num outro contexto, mais esclarecido quanto às problemáticas de se tratar de maneira binômica o "nós e os outros".

Já Guttentag (2009) apresenta um artigo baseado numa revisão da literatura sobre Turismo e Voluntariado que sintetiza os possíveis impactos negativos dessa modalidade de turismo. Os impactos foram separados por tópicos: a) a negligência em relação aos anseios das comunidades hospedeiras; b) a diminuição do progresso dos trabalhos e a realização de trabalhos insatisfatórios; c) a diminuição dos postos de trabalho para a comunidade local e a promoção da dependência; d) a conceitualização do "outro" e a racionalização da pobreza; e) a mudança cultural promovida pelo "efeito demonstração" e pelas missões de curta-duração. 
A respeito da negligência em relação aos anseios das comunidades hospedeiras, o autor salienta que além das motivações altruístas que levam o turista a candidatar-se a trabalhos voluntários, boa parte da motivação é proveniente de razões relacionadas a benefícios pessoais. Se encarado por uma perspectiva estritamente otimista sobre o fenômeno do "Turismo e Voluntariado", tal dado é irrelevante, principalmente quando os intermediadores dos projetos de voluntariado são empresas privadas (as quais, por essência, visam o lucro). Levanta ainda questionamentos, na medida em que os desejos e anseios dos voluntários podem ser colocados à frente das necessidades da população local, buscando a assimilação dos clientes por parte das empresas.

Sobre a diminuição do progresso dos trabalhos e a realização de trabalhos insatisfatórios, Guttentag (2009) relata que esta característica, apesar de ser maléfica para as comunidades que recebem os voluntários, é, também, um dos motivos pelo qual esta modalidade de turismo tem ganhado popularidade. Sob o discurso de "faça o que for possível, mas não deixe de tentar", programas de turismo e trabalho voluntário acabam reunindo uma mão-de-obra desqualificada, motivada pelo "fazer o desenvolvimento". Em virtude dessa desqualificação, trabalhos insatisfatórios e atrasos na conclusão de projetos são resultantes dessa dinâmica, a qual, apesar dos aspectos "práticos" serem negativos, proporcionam uma maior preocupação em relação a determinadas causas (por exemplo, a extinção de tartarugas marinhas, a depredação de um ecossistema, etc.).

Em relação à diminuição dos postos de trabalho para a comunidade local e a promoção da dependência, esse autor discute e critica a visão ingênua sobre o trabalho voluntário e os benefícios socioeconômicos para a população local. Salienta, mediante demonstração de estudos realizados por Van Engen (2000, apud Guttentag 2009), que, pelo fato da grande maioria dos programas de turismo e trabalho voluntário não requisitarem mão-obra qualificada, os voluntários, frequentemente, substituem a mão-de-obra local, causando um desequilíbrio no mercado de trabalho local, pois trabalham de graça (ou pagam para trabalhar). Esse autor também cita Wearing (2001) para ressaltar o aspecto de dependência que o turismo de voluntários pode proporcionar, destaca que o "desenvolvimento vindo de fora" pode induzir a comunidade local a não buscar sua autossuficiência. 
A respeito da conceitualização do "outro" e a racionalização da pobreza, os trabalhos de Simpsom (2004) e Raymond e Hall (2008), como citado por Guttentag (2009), concluem que os estereótipos em relação às comunidades receptoras, ou até mesmo países de forma geral, são reforçados e não esclarecidos, com amplo destaque à "diferença que o voluntário pode fazer com a sua força de vontade". Em relação à racionalização da pobreza, ainda enfatizam que a mensagem "pobre, mas feliz" pode indicar a pobreza como uma "luta que os locais aceitam", quase como um destino.

Sobre a mudança cultural promovida pelo "efeito demonstração" e pelas missões de curtaduração, Guttentag (2009) ainda discute que a interação com a comunidade local e o redirecionamento de divisas do turismo para locais que, em geral, não seriam abordados como destinos turísticos, são considerados como aspectos totalmente positivos do turismo associado ao trabalho voluntário (como o foi em boa parte do corpus da presente pesquisa), Apesar disso, pondera que, o contato com culturas diferentes e com pessoas de níveis socioeconômicos díspares, pode gerar um efeito negativo sobre a comunidade local por meio do "efeito demonstração", que ocorre quando os turistas atraem a atenção para os itens que representam o seu estilo de vida e poder econômico (tais como produtos eletrônicos, roupas de grifes etc.) produzindo na comunidade receptora o desejo de possuir os mesmos padrões de consumo.

Esse autor, porém, não se posiciona contra o citado efeito, uma vez que a exposição a diversos símbolos culturais ocorre de diversas maneiras além do turismo e, por isso, destaca que pode haver consequências positivas, sem, no entanto, citá-las. Apesar de mudanças culturais não intencionais acontecerem (sem julgá-las positivas ou negativas), ressalta que, por vezes, estas podem ser algo desejado por um programa de trabalho voluntariado com turistas como, por exemplo, em expedições de evangelização, também conhecidas como short-term mission trips.

Outros artigos da amostra desta pesquisa relatam diversos possíveis aspectos negativos em relação ao turismo voluntariado: a) Tomazos e Butler (2009b) destacam a perda do caráter altruísta do turismo de trabalho voluntário, tornando-se cada vez mais comercial, intuindo que os anseios do mercado irão se sobrepor aos das comunidades atendidas; b) Palacios (2010) realiza crítica semelhante à de Simpson (2004) e as sintetizadas por Guttentag (2009), 
dissertando sobre como o turismo voluntariado pode se inserir numa óptica neocolonialista, sem o posicionamento da comunidade receptora.

\section{Considerações Finais}

A pesquisa apresentou as abordagens comumente feitas a respeito do tema "Turismo e Voluntariado", destacando artigos de autores internacionais que estudaram a temática em seus artigos publicados em periódicos científicos presentes na WOS e no Scopus. Por meio de filtros de busca, iniciou-se a pesquisa com uma amostra de 155 artigos e chegou-se finalmente a uma amostra de 45 artigos constituindo aproximadamente $30 \%$ de aproveitamento. Essa amostra revelou-se aceitável para o presente estudo, uma vez que reúne artigos que abordam o tema "Turismo e Voluntariado" como tema central e não de forma periférica ou indireta.

Considerando-se que as publicações sobre o tema são recentes, com antecedentes que apontam como marco das investigações o ano de 2000, reforça-se a emergência de um tema, cuja produção científica internacional tem privilegiado os possíveis aspectos positivos que o turismo associado ao trabalho voluntário pode trazer aos envolvidos, supondo-se uma tendência que ocorre quando se trata de práticas de turismo diferentes das "viagens de massa", no âmbito do chamado "turismo alternativo". Além disso, devido ao forte apelo comercial que esta modalidade de turismo apresenta ao aliar "o prazer da viagem ao prazer em ajudar uma causa", é natural que as pesquisas concentrem-se em destacar os possíveis benefícios da prática, sobretudo como maneira de legitimar e ampliar o mercado de viagens com a finalidade de trabalho voluntário aliado ao turismo.

A presente pesquisa, entretanto, também possibilitou explorar estudos que contestam o otimismo exacerbado sobre a prática do turismo associado ao trabalho voluntário, discutindo aspectos que contradizem as primeiras impressões sobre a prática, tais como, o reforço da segregação entre os povos (binômio "desenvolvidos" x "necessitados"), a sobreposição dos anseios dos turistas sobre os interesses da comunidade receptora (intuindo atender e, consequentemente, atrair maior número de voluntários) e a superficialidade das medidas 
voluntárias. Critica-se, também, a escassez de estudos que abordam o turismo associado ao trabalho voluntário sob a perspectiva da comunidade receptora.

Além dos aspectos estritamente relacionados ao conhecimento produzido sobre o tema "Turismo e Voluntariado", a presente pesquisa introduziu uma problemática secundária, a qual não foi aprofundada, porém, afetou o andamento deste estudo: o acesso por via eletrônica às publicações feitas em revistas científicas brasileiras sobre turismo. Destaca-se, além da dificuldade de acesso, que não foram encontrados resultados nas revistas consultadas, o que denota um distanciamento das preocupações da comunidade científica internacional.

Por seu caráter exploratório e qualitativo, a presente pesquisa não é conclusiva, porém, fundamental para melhor posicionamento sobre a questão do turismo associado ao trabalho voluntário. Ressalta-se, ainda, que a temática deste trabalho é ampla e abrange áreas diversas, proporcionando, assim, substrato para entendimento de outros horizontes ligados tanto à temática do trabalho voluntário quanto ao turismo e suas diversificações em termos de novas modalidades de viagens e experiências proporcionadas.

\section{Referências}

AULONG, S.; FIGUIERES, C.; THOYER, S. Agriculture production versus biodiversity protection: The impact of North-South unconditional transfers. Ecological Economics, v. 70, n. 8, pp. 1499-1507, 2011.

BAILEY, A. W.; RUSSELL, K. C. Predictors of interpersonal growth in volunteer tourism: a latent curve approach. Leisure Sciences, v.32, n. 4, pp.352-368, 2010.

BENSON, A. M.; HENDERSON, S. A strategic analysis of volunteer tourism organizations. Service Industries Journal, v.31, n. 3, pp.405-424, 2011.

BLACKMAN, D. A.; BENSON, A. M. The role of the psychological contract in managing research volunteer tourism. Journal of Travel \& Tourism Marketing, v.27, n. 3, pp.221-235, 2010.

BRIGHTSMITH, D. J.; STRONZA, A.; HOLLE, K. Ecotourism, conservation biology, and volunteer tourism: a mutually beneficial triumvirate Biological Conservation, v.141, n.11, pp. 2832- 2842.

BRUMBAUGH, A. M. The impact of diversity seeking and volunteer orientation on desire for alternative spring break programs. Journal of Travel \& Tourism Marketing, v.27, n. 5, pp.474-490, 2010.

CAMPBELL, L.M.; SMITH, C. What makes them pay? Values of volunteer tourists working for sea turtle conservation. Environmental Management, v.38, n.1, pp. 84-98, 2006.

CHEN, Li-Ju; CHEN, J. S. The motivations and expectations of international volunteer tourists: A case study of "Chinese Village Traditions". Tourism Management, v.32, n. 2, pp.435-442, 2011. 
COGHLAN, A. Towards an integrated image-based typology of volunteer tourism organizations. Journal of Sustainable Tourism, v. 15, n.3, pp. 267-287, 2007.

. Exploring the Role of Expedition Staff in Volunteer Tourism. International Journal of Tourism Research, v.10, n.2, pp.183 -191, 2008.

COGHLAN, A.; GOOCH, M. Applying a transformative learning framework to volunteer tourism. Journal of Sustainable Tourism, v. 19, n.6, pp. 713-728, 2011.

FALAGAS, M. E. et al.Comparison of PubMed, Scopus, Web of Science, and Google Scholar: Strengths and weaknesses. The FASEB Journal, v. 22, n. 2, pp. 338-342, 2007. Disponível em: $<$ http://www.fasebj.org/content/22/2/338>, acesso em 2 set. 2011.

GALLEY, G.; CLIFTON, J. The motivational and demographic characteristics of research ecotourists: Operation Wallacea volunteers in South-east Sulawesi, Indonesia. Journal of Ecotourism, v.3, n. 1, pp. 69-82, 2004.

GAP YEAR. What's a Gap Year? Disponível em < http://www.gapyear.com>, acesso em 27 out. 2011.

GRAY, N.J., CAMPBELL, L.M. A decommodified experience? Exploring aesthetic, economic and ethical values for volunteer ecotourism in Costa Rica. Journal of Sustainable Tourism, v. 15, n.5, pp. 463-482, 2007.

GUTTENTAG, D. A, The possible negative impacts of volunteer tourism. International Journal of Tourism Research, v.11, n. 6, pp. 537-551, 2009.

HARLOW, S.; POMFRET, G. Evolving environmental tourism experiences in Zambia. Journal of Ecotourism, v.6, n.3, pp. 184-209, 2007.

HOLMES, K.; SMITH, K. A.; LOCKSTONE-BINNEY, L.; BAUM, T. Developing the dimensions of tourism volunteering. Leisure Sciences, v.32, n.03, pp.255-269, 2010.

HUNTER-JONES, P. The role of charities in social tourism. Current Issues in Tourism, v.14, n.5, pp. 445-458, 2011.

JARVIS, N., BLANK, C. The importance of tourism motivations among sport event volunteers at the 2007 world artistic gymnastics championships, Stuttgart, Germany. Journal of Sport and Tourism, v. 16, n.2, pp. 129-147, 2011.

KEESE, J. R. The Geography of volunteer tourism: Place Matter. Tourism Geographies, v .13, n.02, pp.257-279, 2011.

KENNEDY, K.; DORNAN, D'Arcy. An overview: tourism non-governmental organizations and poverty reduction in developing countries. Asia Pacific Journal of Tourism Research, v.14, n.2, pp.183-200, 2009.

LEE, Y. J.; WOOSNAM, K. M. Voluntourist transformation and the theory of integrative crosscultural adaptation. Annals of Tourism Research, v. 37, n.4, pp. 1186-1189, 2010.

LEPP, A. Leisure and obligation: an investigation of volunteer tourists' experience at Kenya's Taita Discovery Center. Journal of Leisure Research, v.41, n.2, pp. 253-260, 2009.

LO, Ada S.; LEE, Candy Y. S. Motivations and perceived value of volunteer tourists from Hong Kong. Tourism Management, v. 32, n.2, pp.326-334, 2011.

MCGEHEE, N.G. Alternative tourism and social movements. Annals of Tourism Research, v. 29, n. 1, pp. 124- 143, 2002.

MCGEHEE, N. G.; ANDERECK, K. Volunteer tourism and the "voluntoured": the case of Tijuana, Mexico. Journalof Sustainable Tourism, v.17, n.01, pp. 39-51, 2009. 
MCGEHEE, N. G.; SANTOS, C. A.Social change, discourse and volunteer tourism. Annals of Tourism Research, v.32, n.3, pp. 760-779, 2005.

MCINTOSH, A.J.; ZAHRA, A. A cultural encounter through volunteer tourism: Towards the ideals of sustainable tourism? Journal of Sustainable Tourism, v. 15, n.5, pp. 541-556, 2006.

NORUZI, A. Google Scholar: The new generation of citation indexes. International Journal of Libraries and Information Services, v. 55, n. 4, pp. 170-180. 2005. Disponível em:<http://www.librijournal.org/pdf/2005-4pp170-180.pdf>, acesso em nov. 2011.

OOI, N.; LAING, J. H., Backpacker tourism: sustainable and purposeful? Investigating the overlap between backpacker tourism and volunteer tourism motivations. Journal of Sustainable Tourism, v.18, n. 2, pp. 191-206, 2010.

RALSTON, R.; LUMSDON, L.; DOWNWARD, P. The third force in events tourism: Volunteers at the XVII Commonwealth Games. Journal of Sustainable Tourism, v.13, n. 5, pp. 504-519, 2005.

RAYMOND, E. M.; HALL, C. M. The development of cross-cultural (mis)understanding through volunteer tourism. Journal of Sustainable Tourism, v.16, n.5, pp. 530 - 543, 2008.

SIN, Harng Luh, Volunteer tourism "involve me and I will learn"? Annals of Tourism Research, v.36, n.03, pp.480-501, 2009.

. Who are we responsible to? Locals' tales of volunteer tourism. Geoforum, v.41, n.06, pp.983-992, 2010.

THEERAPAPPISIT, P. Pro-poor ethnic tourism in the Mekong: a study of three approaches in Northern Thailand. Asia Pacific Journal of Tourism Research, v.14, n.2, pp. 201-221, 2009.

TOMAZOS, K., BUTLER, R.. Volunteer tourism: The new ecotourism? Anatolia, v. 20, n.01, pp. 196-211. 2009 $\overline{349,2009}$.

. Volunteer tourism: Working on holiday or playing at work?Tourismos, v. 4, n. 04, pp. 331-

. The volunteer tourist as 'hero'. Current Issues in Tourism, v.13, n.04, pp.363-380, 2010.

$\overline{177-187,} 2012$.

Volunteer tourists in the field: A question of balance? Tourism Management, v. 33, n.01, pp.

PALACIOS, C. M. Volunteer tourism, development and education in a postcolonial world: conceiving global connections beyond aid. Journal of Sustainable Tourism,v.18, n.07, pp.861-878, 2010.

PEARCE, D. G. Alternative tourism: concepts, classifications, and questions. In: SMITH, V. L.; EADINGTON, W. R. Tourism alternatives: potentials and problems in the development of tourism.Philadelphia: University of Pennsylvania, pp. 15-30, 1992.

PIOTROWSKA, E. The educational role of youth tourism (on the example of short-time voluntary travel). Prace i Studia Geograficzne, v.33, pp. 225-242, 2003.

SCOPUS. Document search. Disponível em: http://www.scopus.com/home.url. Acesso em out. 2011

SIMPSON, K. 'Doing development': The gap year, volunteer-tourists and a popular practice of development. Journal of International Development, v. 16, n. 5, pp. 681-692. 2004

SINGH, T.V. Altruistic tourism: Another shade of sustainable tourism: The case of Kanda Community. Tourism: An International Interdisciplinary Journal , v. 50, n. 4, pp. 371-381. 2002 
SNYDER, J., DHARAMSI, S., CROOKS, V.A..Fly-By medical care: Conceptualizing the global and local social responsibilities of medical tourists and physician voluntourists. Globalization and Health, v. 7, n. 6. 2011

VODOPIVEC, B., JAFFE, R. Save the world in a week: Volunteer tourism, development and difference. European Journal of Development Research, v. 23, n.1, pp. 111-128. 2011

WEARING, Stephen; NEIL, John.Refiguring self and identity through volunteer tourism. Loisir et Societe, v.23, n. 02, pp. $489-419.2000$

WEB OF SCIENCE. Home. Disponível em:(http://apps.webofknowledge.com/WOS_GeneralSearch_input.do?product=WOS\&SID=1BLgPld PjN19kFm4mPJ\&search_mode=GeneralSearch). Acessado em: out. 2011.

WOOSNAM, K.M., JUNG LEE, Y. Applying social distance to voluntourism research. Annals of Tourism Research, v. 38, n. 1, pp. 309-313. 2011

WYMER Jr., W.W., SELF, D.R., FINDLEY, C.S..Sensation seekers as a target market for volunteer tourism. Services Marketing Quarterly, v. 31, n. 3, pp. 348-362. 2010.

\section{Recebido em: 11/08/2011}

Aprovado em: 07/03/2012 